\title{
SPARING OVARIES
}

\section{SEEMS SAFE}

Ovarian preservation in premenopausal women treated for low-grade, early-stage, endometrial cancer is not associated with increased mortality, say researchers from Columbia University College of Physicians and Surgeons, New York.

Jason D. Wright and colleagues decided to pursue this project because “....we are seeing more and more young women with endometrial cancer, so the question of whether it's safe to preserve the ovaries is coming up more frequently." Potential risks of leaving the ovaries intact include stimulation of residual tumor by ovarian estrogens and coexisting primary ovarian cancer. Nevertheless, only very small reports have been published on the safety of ovarian preservation to date.

Wright et al. used the SEER database to identify a large sample of patients who were representative of women in the US with endometrial cancer. The analysis included 3,269 women aged $\leq 45$ years who were diagnosed with stage I endometrial cancer between 1988 and 2004; 2,867 of these women underwent oophorectomy, whereas the ovaries were spared in the remaining 402 women.

Young age, recent diagnosis, residence in the Eastern US, and low tumor grade were all associated with ovarian preservation. The most important finding of the study was that ovarian preservation had no effect on either disease-specific or overall mortality: the 5-year survival rate for patients with low-grade endometrial cancer was 98\%, regardless of ovarian status.

Given the potential adverse consequences of surgical menopause, such as osteoporosis and cardiovascular disease, Wright et al.'s findings clearly deserve further investigation. Until data from large, prospective studies become available, however, young women with endometrial cancer should be counseled on the possible risks and benefits of ovarian preservation.

\section{Vicky Heath}

Original article Wright, J. D. et al. Safety of ovarian preservation in premenopausal women with endometrial cancer. J. Clin. Oncol. 27, 1214-1219 (2009). 